\title{
BMJ Open Author misrepresentation of institutional affiliations: protocol for an exploratory case study
}

\author{
Vivienne C Bachelet, ${ }^{\odot}$ Francisco A Uribe, Ruben A Díaz, Alonso F Vergara, \\ Fabiana Bravo-Córdova, Víctor A Carrasco, Francisca J Lizana, \\ Nicolás Meza-Ducaud, María S Navarrete
}

\begin{abstract}
To cite: Bachelet VC, Uribe FA, Díaz RA, et al. Author misrepresentation of institutional affiliations: protocol for an exploratory case study. BMJ Open 2019;9:e023983. doi:10.1136/ bmjopen-2018-023983

- Prepublication history for this paper is available online. To view these files, please visit the journal online (http://dx.doi. org/10.1136/bmjopen-2018023983).
\end{abstract}

Received 4 May 2018 Revised 11 November 2018 Accepted 8 January 2019
D) Check for updates

(c) Author(s) (or their employer(s)) 2019. Re-use permitted under CC BY-NC. No commercial re-use. See rights and permissions. Published by BMJ.

Facultad de Ciencias Médicas, Universidad de Santiago de Chile, Santiago, Chile

Correspondence to Dr Vivienne C Bachelet; vivienne.bachelet@usach.cl

\section{ABSTRACT}

Introduction University ranking systems and the publish-or-perish dictum, among other factors, are driving universities and researchers around the world to increase their research productivity. Authors frequently report multiple affiliations in published articles. It is not known if the reported institutional affiliations are real affiliations, which is when the universities have contributed substantially to the research conducted and to the published manuscript. This study aims to establish whether there is an empirical basis for author affiliation misrepresentation in authors with multiple institutional affiliations.

Methods and analysis This individual secondary data exploratory analysis on Scopus-indexed articles for 2016 will search all authors who report multiple institutional affiliations in which at least one of the affiliations is to a Chilean university. We will consider that misrepresentation of an affiliation is more likely when it is not possible to verify objectively a link between the author and the mentioned institution through institutional websites. If we cannot corroborate the author affiliation, we will consider this a finding of potential misrepresentation of the affiliation. We will summarise results with descriptive statistics.

Ethics and dissemination The study protocol was approved by the institutional ethics committee of Universidad de Santiago de Chile, Resolution No. 261, and dated January 15, 2018. Results will be submitted to the World Conference on Research Integrity, among other meetings on publication ethics and research integrity, and will be published in scientific, peer-reviewed journals.

\section{INTRODUCTION}

The push to publish is a well-known and pervasive phenomenon in academia in many different countries. ${ }^{1-5}$ The publish-or-perish dictum puts pressure both on academics and higher education institutions. ${ }^{5-8}$ Academics strive to further their careers, and one of the indicators they are measured by is their research productivity and citations. ${ }^{7910} \mathrm{Simi}-$ larly, universities are interested in bettering their position in the ranking systems, obtaining core and complementary funds
Strengths and limitations of this study

This study introduces the concept of misrepresentation of author institutional affiliation.

- To the best of our knowledge, this will be the first study to explore the prevalence of potential author institutional affiliation misrepresentation.

- Manual data extraction from the Scopus database may increase the risk of measurement error.

- Underestimation or overestimation of study results may occur from information bias.

and complying with local or international accreditation systems. ${ }^{11}$ All of these performance measures rely to an extent on scientific publications-especially those included in the major databases, such as Web of Science or Scopus.

Authorship issues are a central concern in publication ethics ${ }^{12}$ and research integrity. While much has been written about ghost authorship $^{12}$ 13-19 and studies have been conducted on its prevalence, ${ }^{20-25}$ little is known about authors who publish and report multiple institutional affiliations-either university or other-in the author byline. The American Psychological Association (APA) states that "the institutional affiliation identifies the location where the author or authors were when the research was conducted, which is usually an institution." ${ }^{26}$ It then goes on to recommend that a dual affiliation may be included only if 'two institutions contributed substantial support to the study.'

Several factors may contribute to multiple affiliations. ${ }^{27}$ Authors may seek access to resources, networks and infrastructure, on the positive side; or they may be interested in personal financial gain ${ }^{28}$ as may be accrued from universities that pay honoraria to authors to include the university affiliation in the author byline, on the negative side. Accordingly, we could assume that there are 
'legitimate' multiple affiliations-ie, those that comply with the APA guidelines-and 'non-legitimate' multiple affiliations, where at least one of the affiliations is not reflecting a real or substantial contribution by the institution to the study. We understand the latter to be a potential misrepresentation of an institutional affiliation, and this could entail a research integrity breach. However, neither the Committee on Publication Ethics, nor the International Committee of Medical Journal Editors has issued discussion documents or recommendations on the potential ethical implications of affiliation misrepresentation as could occur when the institutions reported have not contributed substantial support to the study.

We believe there may be cases of misrepresentation of affiliations in some articles whose authors report multiple institutional affiliations. Anecdotal verbal reports of misrepresentation of affiliations to Chilean universities have drawn our attention to this possibly emerging misconduct in research integrity. Based on our and other's observations, we know that some authors reporting an affiliation to a Chilean university have received fees for the only purpose of adding on the institutional affiliation, while not having any real academic or research-based employment relationship with the university. The reported institutions of our observations have not contributed any support to the underlying research or the published manuscript.

Chile is a small country with a limited number of universities and stiff competition for prestige and student enrolment. According to the World Bank Country Profile, Chile is a high-income country with a lightly regulated market-based economy, and the private sector is the main provider of higher education. ${ }^{29-31}$ In recent decades, there have been significant efforts to strengthen the quality accreditation process with the purpose of ensuring that minimum quality standards are met. ${ }^{32}$ Universities are classified under three mutually exclusive categories: stateowned and run; private and traditional (founded before 1981); and private non-traditional (founded after 1981). ${ }^{29}$ Profit is not allowed in the Chilean higher education system. ${ }^{29}$ In 2016, there were 62 universities in Chile, ${ }^{29} 52$ of which have at least one article published during 2016 indexed in Scopus. Of these 52 organisations, 16 belong to the state-owned-and-run category, nine are private traditional, and 27 are private non-traditional.

If we are to confirm the emerging problem of institutional misrepresentation of affiliations, the drivers behind it are the need for universities to rise in the international ranking systems for higher education, ${ }^{11} 30$ the linked public funding that is attached to the publications that report university affiliations, ${ }^{29-31}$ and the local quality certification processes. ${ }^{33}$ In other words, we suspect that some institutions might be attempting to game the higher education ranking systems by spuriously pumping up their productivity, while at the same time receiving the benefits of state funding and higher student enrolment. The rise of several non-traditional universities in different rankings in recent years ${ }^{34-38}$ has raised a warning and underpins the need for further research into self-reported institutional affiliations. The consequences of this potential misconduct are important since there would be a violation of public trust, misappropriation of public funds and a distortion of the indicators used by the higher education ranking houses.

The purpose of this exploratory study is to examine and verify affiliations of authors who report multiple affiliations with at least one belonging to a Chilean higher education institution.

\section{METHODS}

\section{Study design}

The design is an individual secondary data exploratory study on Scopus-indexed articles during 2016.

\section{Aims}

Our primary aim is to establish the prevalence of author-reported affiliations to Chilean universities that might potentially misrepresent the true author affiliation based on whether they can be found through a simple Google search. A secondary aim of our study is to determine Open Researcher and Contributor Identification (ORCID) consistency regarding affiliations to Chilean institutions.

\section{Data sources}

We will retrieve all of the articles that have at least one author affiliated to a Chilean university in 2016 as registered by the Scopus database. Scopus is one of the main multidiscipline abstract and citation databases of peer-reviewed literature. It includes scientific journals, books and conference proceedings on research in the fields of science, technology, medicine, social sciences, and arts and humanities. We will use 2016 because this is the most recent year that reasonably includes all articles published during the year in Scopus-indexed scholarly journals.

Affiliations should reflect the contribution that the organisation is giving to the published article, so for this study, we will ascertain whether the reported affiliation is suspect of potential misrepresentation by doing a Google search of the institutional websites, that is, the fact-checking process for this study.

We will also determine the consistency of ORCID author identification database with the affiliation reported in the article. ORCID is a non-profit organisation that helps researchers to be uniquely identified and connected with their contributions and affiliations.

Data for covariables will be obtained from the following: areas of research from Scopus; university profiles from Chilean regulatory documents; and journal impact factor from the Journal Citation Report of Clarivate Analytics.

\section{Search strategy}

To retrieve the articles in Scopus, we developed a specific search strategy for each university, limited to 2016 and document-type 'article'. Table 1 provides a masked 
Table 1 Example of search strategy used in Scopus to find articles with authors affiliated to Chilean universities

\begin{tabular}{ll}
\hline $\begin{array}{l}\text { Name of } \\
\text { university }\end{array}$ & Search strategy \\
\hline Universidad ABCD & AF-ID ('Universidad ABCD' 12345678) \\
& $\begin{array}{l}\text { AND (LIMIT-TO (PUBYEAR, 2016)) AND } \\
\text { (LIMIT-TO (DOCTYPE, 'ar')) }\end{array}$
\end{tabular}

When running all the search strategies, we expect to find over 12 000 articles.

example of the structure of our search strategy, where 'ABCD' can be substituted for the name of any Chilean university. The search strategy also includes a unique Scopus affiliation identifier, also masked in table 1 as ' 12345678 '. The complete Scopus search strategy for all existing Chilean institutions in 2016 is available as supplementary material (available at https://doi.org/10.6084/ m9.figshare.5825943.v1).

\section{Eligibility}

Authors may report single or multiple affiliations. We define 'multiple affiliation' as the reporting of more than one affiliation to organisations involved in researchuniversities, laboratories, commercial research companies, hospitals, non-profit organisations and so forth.

We will include in our study any author who reports in the article author byline an affiliation to a Chilean university, in articles of any field of study. Authors who report an affiliation to a Chilean university may be Chilean, based in Chile; a foreigner, based in Chile; a foreigner, based out of Chile and Chilean based out of Chile. We will not consider the author multiple affiliated if he or she reports different departments belonging to one institution.

\section{Article selection}

We will screen all the eligible records to find authors affiliated with Chilean universities, and we will determine whether they have single or multiple affiliations. Articles that include authors reporting multiple affiliations will be selected for the study.

Seven reviewers working in parallel will retrieve article titles with no duplicate screening of articles for eligibility. Then, we will extract the following data from each article: article title, journal in which the article was published, author's name according to Scopus author detailsthus preventing confusion between authors with similar names-, Chilean university affiliation as it appears in the article Scopus record and author affiliation details according to the Scopus author record.

\section{Data extraction}

For all authors affiliated to a Chilean institution, we will check if those affiliations can be substantiated or not. To do so, we will look into two sources: ORCID database and institutional websites (figure 1). ORCID is a self-reported database, so the authors may not have updated their institutional affiliations, that is, we cannot be sure that the information provided by the authors is complete, updated, transparent or accurate. Likewise, institutional websites might not be updated or might not provide an

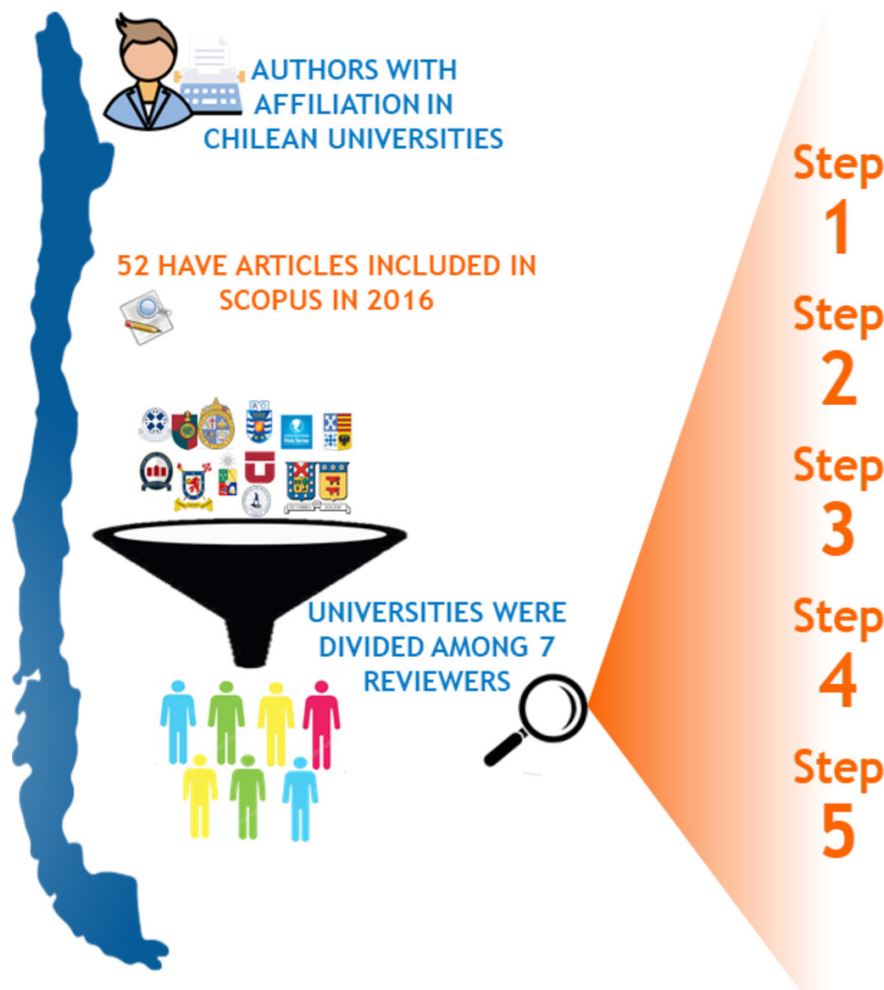

SEARCH IN SCOPUS

2

Step

3

Step

Step
SEARCHIN SCOPUS

FILLING THE DATA EXTRACTION FORM

SINGLE OR MULTIPLE AFFILIATIONS

ORCID INFORMATION

GOOGLE SEARCH
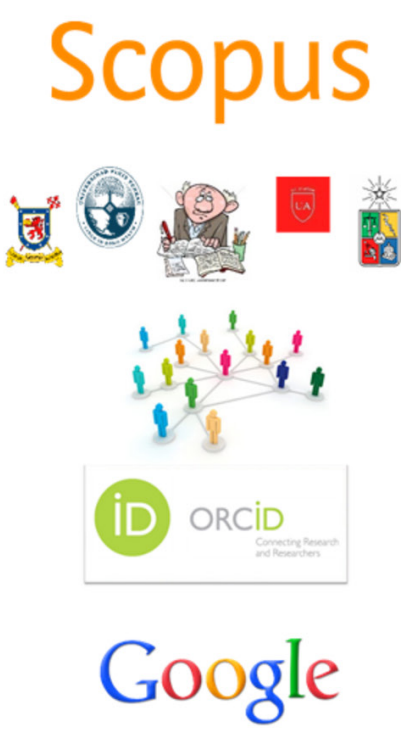

Figure 1 Fact-checking process for potential misrepresentation of institutional affiliations. 
accurate and complete list of affiliated faculties. However, institutional websites are not self-reported by authors, which helps to offset the potential bias in using ORCID.

We will search within the websites of the Chilean universities to find any mention of the authors with affiliations to them. To do this, we will begin by conducting a Google search using the Scopus disambiguated name. We will use the complete disambiguated name; ie, if Scopus reports only the last name and an initial, we will query the Scopus record for the author, and we will look for 'other name formats' to find a complete name that includes last name and first name. We will also add the name of the department or faculty that is being reported in the article to make the Google search more specific. We will set Google to search for results in Chile by default, that is, www. google.cl. We will use this search to verify whether the affiliation reported in the article appears corroborated by the results of the first two Google pages.

We will extract the ORCID id as reported by Scopus, and we will verify whether the author mentions the Chilean institution in his or her ORCID. We will conduct this verification for each unique author who reports an affiliation to a Chilean university in an article indexed in Scopus during 2016. The ORCID author record contains several sections, one of which is 'employment'. We will use this section to verify whether the affiliation reported in the article is also reported in the ORCID author record. If Scopus reports no ORCID for the author, we will hand search the author in ORCID. To hand search in ORCID, we will use the author name as supplied by Scopus. We will screen the first ten records provided by ORCID to find an exact or very close match for the author name, at least to the first and last names. Since ORCID is not disambiguated but instead relies on the author-reported information, more than one author may have the same name. This is a limitation of the ORCID database. To overcome this limitation, we will apply reviewer judgement, discussion and consensus. If the article author affiliation appears in the ORCID record, we will register this information in the data extraction form.

Since our extraction will be done manually, we will introduce quality controls on the data extraction process by conducting crosschecking among the different reviewers. Each reviewer will manually check whether all of the articles resulting from the search strategy assigned to another reviewer were properly extracted into the database. Next, each reviewer will double check each field to detect errors that could arise during data extraction (eg, adding a blank space at the end of a name). After this procedure is completed, a sample will be obtained for each reviewer to measure error rate; if there is at least one error within the sample, the reviewer will have to go through the database again and correct any errors or omissions.

\section{Statistical analysis}

Since there is no prior estimation of the frequency of the event-ie, misrepresentation of author institutional affiliations-we will include the whole population of records available in the Scopus database for 2016, in which at least one author reports at least one affiliation to a Chilean university. Hence, no sampling will be done, and no hypothesis testing is appropriate for these data.

We will consider that potential misrepresentation of affiliation is more likely when it is not possible to objectively verify a link between the author and the mentioned institution. When an author reports the affiliation in ORCID, we will register this finding. A Google search will be conducted to verify whether the affiliation can be substantiated through institutional websites. If we cannot corroborate author affiliation through ORCID and institutional websites, we will consider this to be a finding for potential misrepresentation of the affiliation.

We expect the observed frequency of events to be low and will be reported as a rate. We do not expect to have missing data.

The statistical analysis will be mainly descriptive; the primary objective is to determine the prevalence of potential author institutional affiliation misrepresentation. Exploratory attempts will be made to associate the observed distribution of cases with specific research areas of knowledge (agriculture, energy, health and so on), university profiles (private versus public, traditional versus more recently founded universities and so on), and journal impact factor. We will summarise the findings with descriptive statistics such as means, medians, graphs and tables. We will report the consistency between ORCID self-reporting and corroboration with institutional websites as a proportion. We will report results as per the flowchart shown in figure 2.

\section{Ethics and dissemination}

Due to the possible contentious nature of the matter of this study, we submitted our study protocol to ethical oversight and approval.

Results will be submitted to the World Conference on Research Integrity, among other meetings on publication ethics and research integrity, and will be published in scientific, peer-reviewed journals. The dataset will be openly available at Figshare.

\section{Patient and public involvement}

Neither patients nor public were involved in the development of this study protocol.

\section{DISCUSSION}

We are reporting the protocol for an exploratory study to be conducted in Chile on the potential problem of author affiliation misrepresentation. We expect that the findings of this study might apply to other countries as well. For this reason, we have developed a protocol that could easily be replicated by other investigators. If our working hypothesis is confirmed, this will be the first study to introduce the concept of potential misrepresentation of institutional affiliation and report on its prevalence. Since ORCID is not mandatory, it is also interesting 


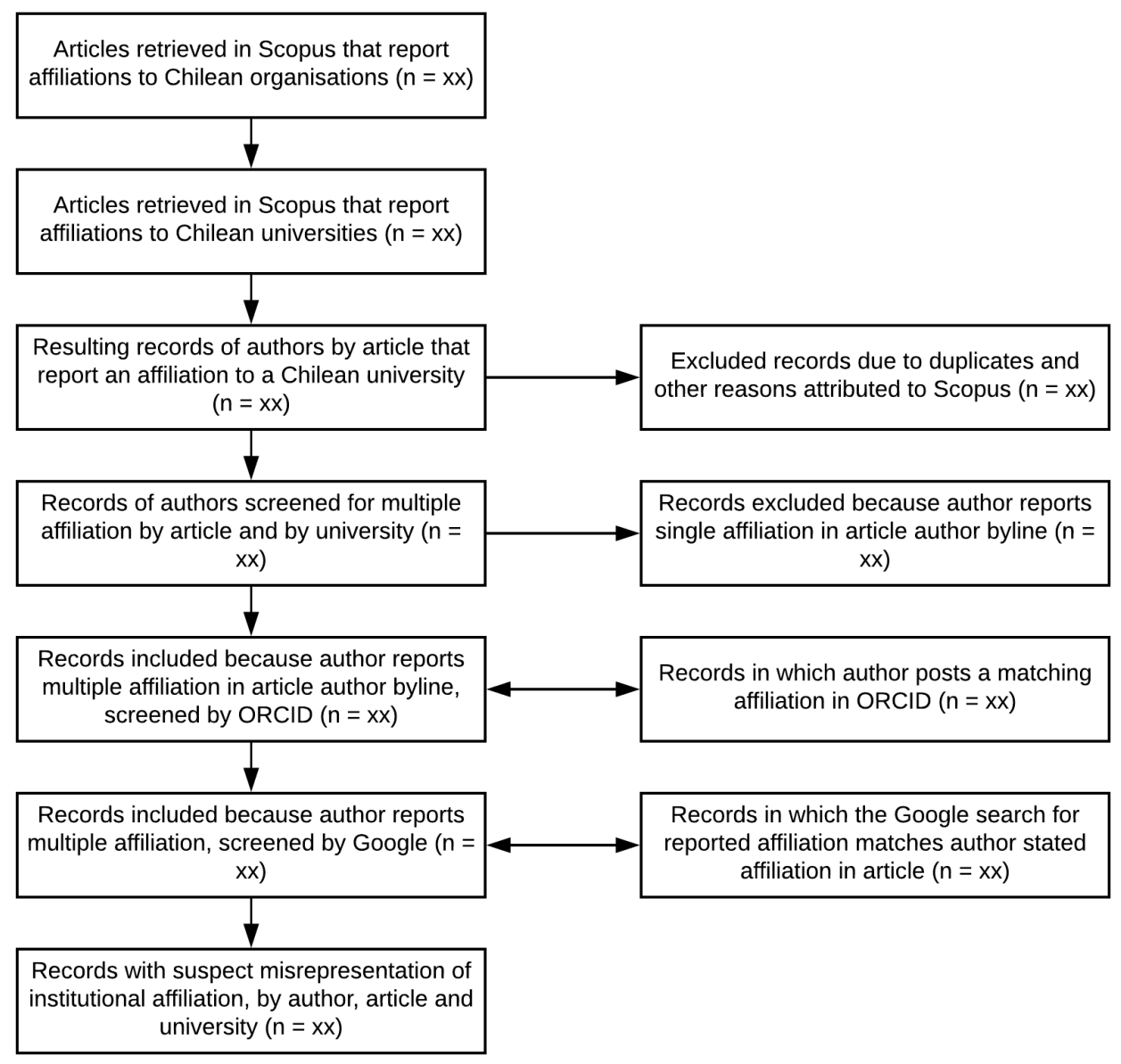

Figure 2 Flowchart of authors reporting Chilean institutional affiliations in Scopus. ORCID, Open Researcher and Contributor Identification.

to assess the penetration of its use among authors reporting an affiliation to a Chilean university, as well as to gauge the consistency between ORCID and institutional websites. Notwithstanding, we are aware that more information is needed on the individual authors to derive any conclusions on potential author misrepresentation of institutional affiliations than what is being collected for this study according to our proposed methods. Certainly no causal relationships can be expected from this study.

Chile has a market-based economy, with a higher education system similar to other countries. In the 2007-2016 period, student enrolment grew at an average annual rate of $5.2 \%$, from 748405 to $1178437 .^{30}$ Universities obtain their funding mainly from enrolment, but they also receive core funding from the state. Core funding in Chile is linked to published papers included in Scopus. ${ }^{30}$ Similarly, research productivity is a key indicator heavily used by most ranking houses. ${ }^{1139}$ Thus, it would not come as a surprise that universities would attempt to recruit highly published and highly cited academics in order to rise in international rankings, a phenomenon that has been reported elsewhere as well. ${ }^{40}$
Few studies have been conducted on the phenomenon of multiple affiliations. One of them showed that multiple affiliations have doubled in recent years in Germany, Japan and the UK. ${ }^{27}$ This may be due to greater international collaboration and globalisation due to modern communications technologies, as well as to increased mobility in academia. Affiliations to different organisations can be construed as facilitating knowledge exchange, without this entailing that there is a misrepresentation of the affiliation. Universities should pay authors for their work, but there may also be secondary appointments that include visiting positions, courtesy appointments or emeritus status, among others. These secondary or honorary appointments may enable and further facilitate continued collaboration between researchers, with no additional commitment for space or resources on the part of the institution. Some institutions have complete descriptions of the privileges and responsibilities of these secondary appointments. Since there are no consensus-based guidelines on what defines an author affiliation and, to our best knowledge, there is none beyond the APA definition, we believe there is room for manipulation of this key information during the process of article 
submission for unethical ends. In effect, further research is needed to determine the true underlying causes for the increase of multiple affiliations.

Institutional author affiliation misrepresentation, if ascertained, is a complex problem with many potential causes, some of which may be context specific, and international drivers such as ranking and academic compensation systems may explain others. In our study, we have strived to design a protocol that accounts for the most commonly identified uses for author affiliations and that are present in all countries with robust university systems.

\section{Strengths and limitations}

We have thoughtfully considered the difficulties in tying an investigator's name to a supporting institution, especially considering that many times universities are not prompt at disclosing on their websites who is affiliated to them. Nonetheless, we believe this is an institutional mandate that world-class universities, that is, those that seek to appear in the ranking systems, must comply with. Accordingly, the study group chose an approach that we believe is one of the strengths of this study, which is to corroborate author institutional linkage with third-party entities-ORCID and institutional websites-thus minimising the risk of information bias and methodological variability.

Robustness of our data depends greatly on the soundness of the individual Scopus author and article records, which are not self-reported. This is a strength of the study, because we are using a standardised and internationally recognised data source for scientific productivity, but it is also a limitation, as there could be potential errors in the Scopus dataset that we would not be able to correct for. ${ }^{41}$ Nevertheless, Scopus disambiguates author names in the Scopus Author Profile, which is automatically created by Scopus using a sophisticated algorithmic profiling that Scopus itself admits is not $100 \%$ accurate. ${ }^{42}$ Using the data extracted, we will be able to explore associations with journals (including journal disciplinary category and impact factor), author countries of origin and category of university that the suspect affiliation is reporting, among others. This will provide us with a full characterisation and scope of author multiple affiliations that include affiliations to Chilean universities. Exploring the whole dataset of authors reporting an affiliation to a Chilean institution in a given year instead of resorting to a sampling strategy is, in our view, a strength of this study, but the manual extraction of data from the Scopus website is a limitation. This could be offset by an electronic export of the Scopus database thus helping reduce measurement error.

Another strength of this study is that we are using internationally established standards to corroborate institutional affiliations, such as ORCID and Google, present in most countries. This study will also help to provide numbers for the penetration of ORCID with authors reporting an affiliation to a Chilean higher education institution. While Scopus author records are not self-reported, ORCID is self-reported, making the corroboration of institutional affiliation through ORCID susceptible to information bias. Conversely, institutional websites are not self-reported but may not be updated. Combining these multiple checkpoints should make our conclusions more robust.

This study has many limitations that cannot be corrected for by method. For example, we will not able to corroborate whether an author is using a single affiliation that is misrepresented versus multiple affiliations that might or might not be misrepresented. The former could be the case if an author publishes an article on behalf of an institution while not being a member of faculty or having any real linkage to the university.

We hope that our study results will contribute to raise awareness and guide key stakeholders in developing standards for reporting institutional affiliations. Affiliations should truly reflect the support and contributions provided to the research and the publication of the study results by the universities mentioned in the author byline. If we validate the existence of author and institutional misconduct in the case of Chile and applying the methodology that this study will pilot, we are interested in conducting a larger more comprehensive study that would include a panel of countries to represent both emerging economies and developed countries.

Acknowledgements The authors wish to thank Eva Madrid (Universidad de Valparaíso, Chile), Adrian Ziderman (Bar-llan University, Israel) and Miguel Roig (St. John's University, United States) for providing helpful ideas, guidance and comments.

Contributors Study idea (VCB); conceptualisation (VCB, FAU, RAD, AFV, MSN, FBC, VAC, FJL, NMD); methodology (VCB, FAU, RAD, AFV, MSN); original draft preparation (VCB, FAU); review and editing (VCB, FAU, RAD, AFV, MSN, FBC, VAC, FJL, NMD); supervision (VCB, FAU); project administration (VCB).

Funding The authors have not declared a specific grant for this research from any funding agency in the public, commercial or not-for-profit sectors.

Competing interests All authors are affiliated to a state university and this might be construed as a potential bias regarding the research protocol reported. The lead author (VCB) is member of the Committee on Publication Ethics council.

\section{Patient consent for publication Not required.}

Ethics approval The institutional ethics committee of Universidad de Santiago de Chile, Resolution No. 261, dated 15 January 2018, approved the study protocol

Provenance and peer review Not commissioned; externally peer reviewed.

Open access This is an open access article distributed in accordance with the Creative Commons Attribution Non Commercial (CC BY-NC 4.0) license, which permits others to distribute, remix, adapt, build upon this work non-commercially, and license their derivative works on different terms, provided the original work is properly cited, appropriate credit is given, any changes made indicated, and the use is non-commercial. See: http://creativecommons.org/licenses/by-nc/4.0/.

\section{REFERENCES}

1. Mandal M, Bagchi D, Basu SR. Scientific misconducts and authorship conflicts: Indian perspective. Indian J Anaesth 2015;59:400-5.

2. Glezerman M, Grossman E. [Scientific publications: on honors and ghosts]. Harefuah 2017;156:365-8.

3. Mubeen SM, Shaikh MA. Publish an original article or perish: only way to promote research in Pakistan? J Pak Med Assoc 2017;67:154-5

4. Grančay M, Vveinhardt J, Šumilo Ērika. Publish or perish: how Central and Eastern European economists have dealt with the 
ever-increasing academic publishing requirements 2000-2015. Scientometrics 2017;111:1813-37.

5. Chou CP, Chan C-F. Trends in publication in the race for worldclass University: the case of Taiwan. Higher Education Policy 2016;29:431-49.

6. Hangel N, Schmidt-Pfister D. Why do you publish? On the tensions between generating scientific knowledge and publication pressure. Aslib Journal of Information Management 2017;69:529-44.

7. Hasan SS, Ahmadi K. Publish or Perish: a mandate with negative collateral consequences. Acad Med J Assoc Am Med Coll 2017;92:140.

8. Génova G, de la Vara JL. The problem is not professional publishing, but the publish-or-perish culture. Sci Eng Ethics 2018.

9. Davies B, Felappi G. Publish or Perish. Metaphilosophy 2017:48:745-61.

10. Weisshaar K. Publish and Perish? An assessment of gender gaps in promotion to tenure in Academia. Social Forces 2017;96:529-60.

11. Marope PTM, Wells PJ, Hazelkorn E. Rankings and accountability in higher education: uses and misuses. France: UNESCO Publishing, 2013.

12. COPE Council. What constitutes authorship? COPE Discussion document. 2014 http://publicationethics.org/files/Authorship_ DiscussionDocument.pdf

13. Gasparyan AY, Ayvazyan L, Kitas GD. Authorship problems in scholarly journals: considerations for authors, peer reviewers and editors. Rheumatol Int 2013;33:277-84

14. Moher D. Along with the privilege of authorship come important responsibilities. BMC Med 2014;12:214.

15. Emeson U, Pugh DM, Scruth EA. Ethical and legal aspects of inappropriate (ghost) authorship. Clin Nurse Spec 2015;29:131-3.

16. Hamilton CW, Donnelly J, Gertel A, et al. Ghost authorship. Home Healthc Now 2015;33:346.

17. Anthony M. Honorary and ghost authorship. Home Healthc Now 2015;33:125-6.

18. Kornhaber RA, McLean LM, Baber RJ. Ongoing ethical issues concerning authorship in biomedical journals: an integrative review. Int J Nanomedicine 2015;10:4837-46.

19. Teixeira da Silva JA, Dobránszki J. Multiple Authorship in Scientific Manuscripts: Ethical Challenges, Ghost and Guest/Gift Authorship, and the Cultural/Disciplinary Perspective. Sci Eng Ethics 2016;22:1457-72.

20. Flanagin A, Carey LA, Fontanarosa PB, et al. Prevalence of articles with honorary authors and ghost authors in peer-reviewed medical journals. JAMA 1998;280:222-4.

21. Wislar JS, Flanagin A, Fontanarosa PB, et al. Honorary and ghost authorship in high impact biomedical journals: a cross sectional survey. BMJ 2011;343:d6128.

22. Dotson B, Slaughter RL. Prevalence of articles with honorary and ghost authors in three pharmacy journals. Am J Health Syst Pharm 2011:68:1730-4.

23. Mirzazadeh A, Navadeh S, Rokni M, et al. The prevalence of honorary and ghost authorships in Iranian bio-medical journals and its associated factors. Iran J Public Health 2011;40:15-21.
24. Kennedy MS, Barnsteiner J, Daly J. Honorary and ghost authorship in nursing publications. J Nurs Scholarsh 2014;46:416-22.

25. Vera-Badillo FE, Napoleone M, Krzyzanowska MK, et al. Honorary and ghost authorship in reports of randomised clinical trials in oncology. Eur J Cancer 2016;66:1-8.

26. American Psychological Association. APA. 6th edn, 2010.

27. Hottenrott $\mathrm{H}$, Lawson $\mathrm{C}$. A first look at multiple institutional affiliations: a study of authors in Germany, Japan and the UK. Scientometrics 2017;111:285-95.

28. Stephan P. How economics shapes science. Harvard University Press 2015.

29. Contraloría General de la República. Financiamiento fiscal a la educación superior 2016. Santiago, 2017.

30. Galleguillos P, Hernández T, Sepúlveda F, et al. Reforma a la educación superior: Financiamiento actual y proyecciones. Santiago, 2016.

31. Consorcio de Universidades del Estado de Chile. Análisis del Financiamiento Fiscal a la Educación Superior Años 2011 a 2014. Santiago, 2016.

32. Comisión de Acreditación de Chile. Guía para la evaluación externa. https://www.cnachile.cl/Documentos\%20de\%20Paginas/Gu\%C3\% ADa\%20para\%20la\%20Evaluaci\%C3\%B3n\%20Externa.pdf

33. Zapata G, Clasing P. El uso de criterios e indicadores de calidad en la acreditación de programas: diferencias entre agencias privadas de acreditación en Chile. 2016 https://doc-10-bs-appsviewer.googleusercontent.com/viewer/secure/pdf/risup52gtdie tt753a2cnd1s1 qjjgrtf/rg8dh9u1dhtam67ushipe3hcdpimoprj/ 1503606825000/gmail/12817934390026887180/ACFrOgAi_KjIS VnW5rGV08tNy9YIZKq2xaz6AMIBCgqe_SHcdIIWeE1U_LIPAQwuH OKJad4jNJTIw8

34. El Most. U. Autónoma de Chile dentro de las 70 mejores universidades de América Latina. 2017 http://www.elmostrador.cl/ agenda-pais/vida-en-linea/2017/07/26/u-autonoma-de-chile-dentrode-las-70-mejores-universidades-de-america-latina/

35. La Tercera. UDP se posiciona como la mejor universidad joven de América Latina. 2017 http://www2.latercera.com/noticia/udp-seposiciona-la-mejor-universidad-joven-america-latina/\#

36. Monzón C. Ranking de Shanghai: dos universidades chilenas entre las 500 mejores del mundo. Publimetro. $2017 \mathrm{https}: / / \mathrm{www}$. publimetro.cl/cl/noticias/2017/08/15/ranking-shanghai-dosuniversidades-chilenas-las-500-mejores-del-mundo.html

37. Emol. U. Federico Santa Maria y UC son las mejores universidades chilenas en ranking internacional. 2016.

38. INTRANET. UNAB se ubica dentro de las tres mejores universidades del país en ranking SCImago. 2017.

39. Aguillo IF, Bar-llan J, Levene M, et al. Comparing university rankings. Scientometrics 2010;85:243-56.

40. Bhattacharjee Y. Citation impact. Saudi universities offer cash in exchange for academic prestige. Science 2011;334:1344-5.

41. Schmidt CM, Cox R, Fial AV, et al. Gaps in affiliation indexing in Scopus and PubMed. J Med Libr Assoc 2016;104:138-42.

42. Scopus. Author Identifiers. 2018 https://blog.scopus.com/topics/ author-identifiers 